\title{
Study of monolayer polymerization using nonlinear optics
}

\author{
G. Berkovic, Th. Rasing, and Y. R. Shen \\ Department of Physics, University of California, Center for Advanced Materials, Lawrence Berkeley \\ Laboratory, Berkeley, California 94720
}

(Received 10 July 1986; accepted 9 September 1986)

\begin{abstract}
Optical second harmonic generation has been used to study the polymerization of a monolayer of vinyl stearate or octadecyl methacrylate spread at the air/water interface. Rapid polymerization occurred when monolayers were irradiated with ultraviolet light, while some slower polymerization occurred when certain redox initiators were added to the water subphase. Information regarding polymerization kinetics and the orientation of molecules at the interface were also obtained using this method.
\end{abstract}

\section{INTRODUCTION}

Polymerization in monomolecular layers has attracted widespread interest in both basic and applied research. As well as being employed in microlithography, ${ }^{1}$ microelectronics, ${ }^{2}$ and synthetic lipids, ${ }^{3}$ monolayer polymerizations are of fundamental interest since their reactivity and kinetics may be studied under controllable and variable conditions of molecular separation and orientation. ${ }^{4}$

Unfortunately, such studies have been limited due to a lack of techniques sufficiently sensitive and selective to probe adsorbate monolayers on surfaces. However, it has been demonstrated in recent years that nonlinear optical techniques such as second harmonic generation (SHG) are particularly suitable in this regard. ${ }^{5,6}$ The applicability of SHG arises from its submonolayer sensitivity and high surface specificity for adsorbates on isotropic or centrosymmetric substrates.

Second harmonic generation arises from a nonlinear polarization $P$ induced in a medium at frequency $2 \omega$, given by ${ }^{5}$

$$
\mathbf{P}(2 \omega)=\chi^{(2)} \mathbf{E}(\omega) \mathbf{E}(\omega),
$$

where $\chi^{(2)}$ is a second order nonlinear susceptibility. The signal strength is generally proportional to $|P(2 \omega)|^{2}$. The surface specificity of SHG is a result of the symmetry properties of $\chi^{(2)}$ which vanishes (under the electric dipole approximation) for a centrosymmetric medium. Since inversion symmetry is broken at an interface, $\chi^{(2)} \neq 0$ and an SHG signal can be expected to originate from the surface layer. ${ }^{5}$ Thus, SHG can be used to study adsorbates on a substrate.

We report here a study, using SHG, of monolayer polymerization at an air-water interface. We have studied two materials (see Fig. 1), vinyl stearate (VS) and octadecyl methacrylate (ODMA). They were chosen because of their ability to spread as monolayers on water, and to polymerize upon irradiation with ultraviolet light. ${ }^{4,7-11}$ Apart from the inherent surface specificity of SHG, this technique has an additional advantage in the present experiment; namely that it can be performed directly and nondestructively at the airwater interface. This is in contrast to earlier infrared studies of these systems, ${ }^{12-14}$ in which the monolayers had to be transferred onto a suitable solid substrate for analysis.

\section{EXPERIMENTAL}

The materials used in this study were of commercial origin. Vinyl stearate (Chemtech Research Inc.) and octadecyl methacrylate (Sartomer) monomers were both purified according to published methods. ${ }^{11,15}$ Commercial samples of the polymers of these materials (Aldrich) were used without further purification.

The experimental apparatus has been described elsewhere ${ }^{16}$; it consists of focusing the frequency doubled output $(532 \mathrm{~nm})$ of a Q-switched, mode-locked Nd-YAG laser onto a Langmuir trough on which the monolayer is spread, and detecting the $266 \mathrm{~nm}$ photons in the reflected ouput. Since the average number of photon counts detected per laser pulse was less than one, the signal was averaged over at least $4 \times 10^{4}$ pulses.

Monolayers were spread by pipetting an appropriate volume of a petrol ether solution and allowing the solvent to evaporate. It was verified that the solvent left no residue causing additional SHG.

Samples were polymerized by placing a Pen Ray ultraviolet source (Ultraviolet Products Inc., San Gabriel, CA) approximately $15 \mathrm{~cm}$ above the water surface. During irradiation the monolayer area was kept constant and the Langmuir trough was enclosed in a Plexiglass box and kept under nitrogen. The far UV intensity on the water surface was estimated to be $40 \mu \mathrm{W} / \mathrm{cm}^{2}$.

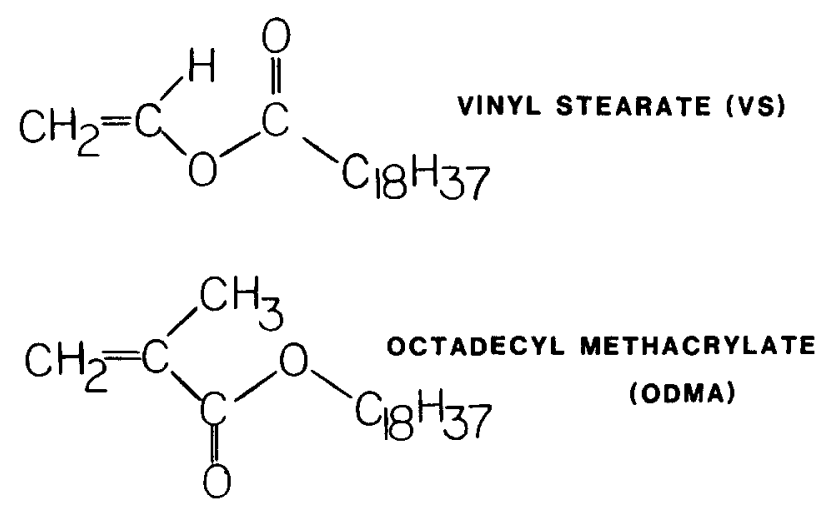

FIG. 1. Monomers used in this study. 
All experiments were performed at room temperature $\left(20 \pm 1^{\circ} \mathrm{C}\right)$.

\section{RESULTS AND DISCUSSION}

As a first step we compared the SHG signals from pure water and VS monomer and polymer monolayers on water. The number of second harmonic photons detected per unit time $S$ is given by

$$
S \propto\left|\chi^{(2)}\right|^{2},
$$

where $\chi^{(2)}$ is the second order nonlinear susceptibility. A pure water surface has a nonnegligible susceptibility $\chi_{w}$ while for an adsorbate covered surface $\chi^{(2)}=\chi_{\omega}^{(2)}+\chi_{s}^{(2)}$, where $\chi_{s}^{(2)}$ is the susceptibility of the adsorbate layer. ${ }^{17}$

Vinyl stearate was spread on water to give a surface density of $27 \AA^{2} /$ molecule. This surface density, which is about $30 \%$ less dense than that of a close packed monolayer, leads to the most efficient polymerization at constant area due to the lack of steric hinderance between adjacent monomers. ${ }^{4.18}$ As shown in Table I, the second harmonic intensity generated from this surface was about 2.6 times that of the pure water surface. For a monolayer of authentic poly-VS at the same surface area per monomeric unit, the SHG was 1.8 times that of water. Thus, the SHG signal clearly differentiates between a pure water surface, and monomer and polymer monolayers on water. Furthermore, after the monomer monolayer was irradiated by ultraviolet light for $2 \mathrm{~h}$, the SHG signal became very close to that of the authentic polymer monolayer, suggesting that essentially complete polymerization had taken place.

Table I also shows a similar observation for ODMA. The signals from a monolayer of monomer and polymer molecules are clearly different, and after UV irradiation of the monomer monolayer, the SHG signal closely corresponds to that of the polymer monolayer.

We also note here that although the incident laser radiation at $532 \mathrm{~nm}$ was intense enough to enable observable SHG, it was insufficient to initiate polymerization via multiphoton absorption. This was deduced from the fact that the

TABLE I. Relative intensities of second harmonic generation from a water surface covered with various monolayers. All experiments were performed as follows: Input angle of incidence $=60^{\circ}$; input laser polarization $=45^{\circ}$ from plane of incidence. (The SHG intensities in this table represent total SHG detected, i.e., the output was not resolved into polarization components.)

\begin{tabular}{cc}
\hline Monolayer & Relative SHG intensity \\
\hline Water only & 100 \\
VS $^{\mathrm{a}}\left(27 \AA^{2} /\right.$ molecule $)$ & 260 \\
Poly VS $\left(27 \AA^{2} /\right.$ monomeric unit $)$ & 170 \\
VS monolayer after UV irradiation & 180 \\
& \\
Water only & 100 \\
ODMA $^{\mathrm{b}}\left(26 \AA^{2} /\right.$ molecule $)$ & 370 \\
Poly ODMA (26 $\AA^{2} /$ monomeric unit) & 220 \\
Poly ODMA after UV irradiation & 250 \\
\hline
\end{tabular}

a $\mathrm{VS}=$ vinyl stearate.

${ }^{\mathrm{b}}$ ODMA = octadecyl methacrylate.
SHG signal from monomer monolayers was unchanged during prolonged laser irradiation.

The observation that, in our case, SHG from monomers is larger than that of their corresponding polymers can be understood as follows: Second order optical nonlinearity of molecules arises mainly from chemical bonds in which the electron distributions are more readily distorted by optical excitation. ${ }^{19}$ In VS and ODMA the $\pi$ electrons in the double bonds are likely to dominate the nonlinearity. Since the polymerization process breaks a carbon-carbon double bond the optical nonlinearity decreases. It thus may also be expected that SHG from bulk and surface polymerized samples of the same polymer should be essentially the same, although their molecular weight distributions may be different.

The kinetics of the polymerization could be determined by monitoring the second harmonic signal $S$ as the reaction proceeded (see Fig. 2). For kinetic analysis, a quantity proportional to $\theta$, the fraction of unreacted monomers, must be extracted from the data. This is done as follows:

$$
\begin{aligned}
S^{1 / 2} \propto \chi^{(2)} & =\chi_{w}^{(2)}+\chi_{s}^{(2)} \\
& =\chi_{w}^{(2)}+\theta \chi_{m}^{(2)}+(1-\theta) \chi_{p}^{(2)},
\end{aligned}
$$

where $\chi_{m}^{(2)}$ and $\chi_{p}^{(2)}$ are the second order nonlinear susceptibilities of a full monolayer of monomer and polymer, respectively. After some simple algebra, we find

$$
\theta(t) \propto \chi^{(2)}(t)-\chi^{(2)}(\infty)=S^{1 / 2}(t)-S^{1 / 2}(\infty),
$$

where $S(\infty)$ and $\chi^{(2)}(\infty)$ are the SHG signal and the susceptibility at $t \rightarrow \infty$ when the polymerization is complete.

The polymerization should follow some rate law:

$$
\frac{d \theta}{d t}=-k \theta^{n} \text {. }
$$

For this type of polymerization, both second order ${ }^{4}$ $\left(\theta^{-1} \propto t\right)$ and first order ${ }^{10}(\ln \theta \propto t)$ kinetics have been proposed; the different rate laws may arise if during the reaction the concentration of reactive radicals is proportional to $\theta$, or quasiconstant, as a result of various chain termination processes. ${ }^{20} \mathrm{~A}$ kinetic equation where $n=3 / 2\left(\theta^{-1 / 2} \propto t\right)$ is also possible. ${ }^{20}$ In all cases, the best fit to the kinetics should be found in the initial stages of the reaction, since some deviation may occur as the reaction nears completion due to the entrapment of unreacted monomers inside polymer chains, and a large increase in surface viscosity ${ }^{10}$ when polymerization is nearly complete.

Figure 2 shows the raw data $S(t)$ as well as attempts to fit the data using Eqs. (4) and (5). Both $n=1$ and $n=2$ in Eq. (5) appear to satisfactorily describe the experimental results. Thus the data are not accurate enough for us to clarify the kinetic behavior. This question should be more easily resolvable in a system exhibiting a larger change in SHG during polymerization.

It has also been reported that monolayer polymerization of vinyl stearate may be accomplished by addition of a redox initiator $\left(\mathrm{NH}_{4}\right)_{2} \mathrm{~S}_{2} \mathrm{O}_{8} / \mathrm{NaHSO}_{3}{ }^{7}$ or $\mathrm{K}_{2} \mathrm{~S}_{2} \mathrm{O}_{8}{ }^{18}$ to the water subphase. However, the two reports differ markedly with respect to the polymerization time. The first report, ${ }^{7}$ which used the mixed initiator and monitored the surface pressure during reaction, claimed that polymerization was complete 


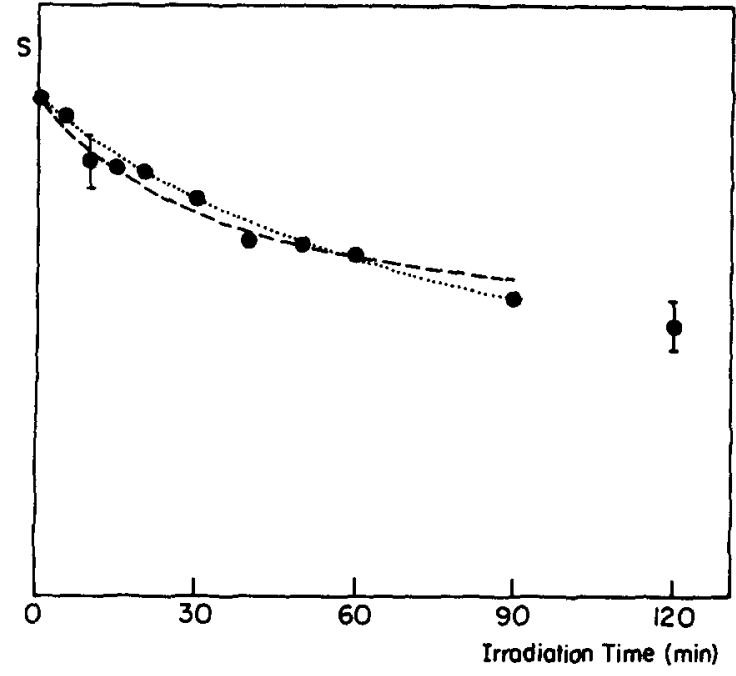

FIG. 2. The relative SHG intensity $(S)$ is plotted against irradiation time for UV polymerization of ODMA. The data (O) can be fitted satisfactorily by both first order $(\cdots)$ and second order $(---)$ kinetics (see the text). All experimental data points have the same uncertainty, although error bars for most points have been omitted for clarity.

within $1 \mathrm{~h}$. The second report, ${ }^{18}$ using only $\mathrm{K}_{2} \mathrm{~S}_{2} \mathrm{O}_{8}$ as initiator and monitoring surface viscosity, found a reaction rate slower by about two orders of magnitude. We have repeated these measurements using SHG as the probe. We observed that using either initiator system, the SHG signal decreased by not more than $10 \%$ from the monomer value over a period of $4 \mathrm{~h}$, indicating a very slow reaction, in better agreement with the rate reported in the second publication. ${ }^{18}$

It has been shown $n^{5}$ that in certain cases, the polarization dependence of the SHG signal can be used to determine the average orientation of molecules at interfaces. We found, in our case, that both monomers generated a second harmonic signal having the $s$-polarized component stronger (by a factor of about 2) than the $p$-polarized component. However, SHG from both polymers was $p$ polarized. As shown earlier, ${ }^{5}$ it is only possible to deduce average molecular orientations from SHG if the nonlinear susceptibility tensor is dominated by a single component

$$
\chi_{s}^{(2)}=N\left\langle\alpha_{\zeta 55}^{(2)}\right\rangle \text {, }
$$

where $\alpha^{(2)}$ is the nonlinear polarizability of a single molecule, $\zeta$ is a molecular axis, $N$ is the number of molecules per unit surface area, and the brackets signify an average over molecular orientations.

Since the molecular nonlinearity arises mainly from the $\pi$ electrons, ${ }^{19}$ the spatial directions of the $\pi$ bonds should determine the polarization of the generated second harmonic radiation. Unfortunately since each monomer has two $\pi$ bonds whose spatial orientations are independent of one another, there is no unequivocable transformation from the observed SH polarization to a molecular orientation at the surface. However, for the polymer, the only $\pi$ electrons are found in the $\mathrm{C}=\mathrm{O}$ bond and the observed $p$-polarization of the SH signal suggests that the $\mathrm{C}=\mathrm{O}$ bond is directed perpendicular to the water surface. This conclusion is in agreement with both theoretical predictions ${ }^{11}$ and analysis of polarized IR spectra ${ }^{12-14}$ of Langmuir-Blodgett monolayers and multilayers of these systems.

In conclusion, we have shown that optical SHG is a viable technique to study a chemical reaction like polymerization on a monolayer scale. This technique enables us to follow the kinetics of the reaction and also yields information about the orientation of molecules at the interface. As we have demonstrated, this method is applicable even in the case of polymerization of simple vinyl and methacrylate monomers, whose optical nonlinearity is very small (comparable to that of the water surface).

\section{ACKNOWLEDGMENTS}

This work was supported by the Director, Office of Energy Research, Office of Basic Energy Sciences, Material Sciences Division of the U. S. Department of Energy under Contract No. DE-AC03-76SF00098. We thank the Sartomer Co. for a gift of octadecyl methacrylate. G. B. acknowledges the financial support of a Chaim Weizmann Postdoctoral Fellowship.

'A. Barraud, C. Rosilio, and R. Ruaudel-Teixier, Thin Solid Films 68, 91 (1980).

${ }^{2}$ G. L. Larkins, Jr., E. D. Thompson, M. J. Deen, C. W. Burkhart, and J. B. Lando, IEEE Trans. Magn. 19, 980 (1983).

${ }^{3}$ R. Elbert, A. Laschewsky, and H. Ringsdorf, J. Am. Chem. Soc. 107, 4134 (1985).

${ }^{4}$ S. A. Letts, T. Fort, Jr., and J. B. Lando, J. Colloid Int. Sci. 56, 64 (1976).

${ }^{5}$ H. W. K. Tom, T. F. Heinz, and Y. R. Shen, Laser Chem. 3, 279 (1983).

${ }^{6}$ Y. R. Shen, J. Vac. Sci. Technol. B 3, 1464 (1985).

'H. Z. Friedlander, U. S. Patent 3,031,721 (1962).

${ }^{8}$ K. C. O'Brien, J. Long, and J. B. Lando, Langmuir 1, 514 (1985).

${ }^{9}$ N. Beredjick and W. J. Burlant, J. Polym. Sci. Part A1 8, 2807 (1970).

${ }^{10}$ A. Dubault, C. Casagrande, and M. Veyssie, J. Phys. Chem. 79, 2254 (1975).

"D. Naegele and H. Ringsdorf, J. Polym. Sci. Polym. Chem. Ed. 15, 2821 (1977).

${ }^{12}$ V. Enkelmann and J. B. Lando, J. Polym. Sci. Polym. Chem. Ed. 15, 1843 (1977).

${ }^{13} \mathrm{~K}$. Fukuda and T. Shiozawa, Thin Solid Films 68, 55 (1980).

${ }^{14}$ S. J. Mumby, J. D. Swalen, and J. F. Rabolt, Macromolecules 19, 1054 (1986).

${ }^{15}$ W. Burlant and A. Adicoff, J. Polym. Sci. 27, 269 (1958).

${ }^{16}$ Th. Rasing, G. Berkovic, Y. R. Shen, S. Grubb, and M. W. Kim, Chem. Phys. Lett. 130, 1 (1986).

${ }^{17}$ Th. Rasing, Y. R. Shen, M. W. Kim, P. Valint, Jr., and J. Bock, Phys. Rev. A 31, 537 (1985).

${ }^{18}$ K. Fukuda, Y. Shibasaki, and H. Nakahara, Chem. Phys. Chem. Anwendungstech. Grenzflachenakt. Stoffe; Ber. Int. Kong. VI (Zurich, 1972) Band 2,1 p. 273.

${ }^{19}$ D. J. Williams, Angew. Chem. Int. Ed. 23, 690 (1984).

${ }^{20} \mathrm{P}$. J. Flory, Principles of Polymer Chemistry (Cornell University, Ithaca, 1953), pp. 110-115. 